\title{
ON LEAST SQUARES DISCRETE FOURIER ANALYSIS OF UNEQUALLY SPACED DATA
}

Abstract. The problem of discrete Fourier analysis of observations at nonequidistant times using the standard set of complex harmonics $\exp (i 2 \pi k t)$, $t \in \mathbb{R}, k=0, \pm 1, \pm 2, \ldots$, and the least squares method is studied. The observation model $y_{j}=f\left(t_{j}\right)+\eta_{j}, j=1, \ldots, n$, is considered for $f \in L^{2}[0,1]$, where $t_{j} \in[(j-1) / n, j / n)$, and $\eta_{j}$ are correlated complex valued random variables with $E_{\eta} \eta_{j}=0$ and $E_{\eta}\left|\eta_{j}\right|^{2}=\sigma_{\eta}^{2}<\infty$. Uniqueness and finite sample properties of the observed function Fourier coefficient estimators $\hat{c}_{k}, k=$ $0, \pm 1, \ldots, \pm m$, where $m<n /(8 \pi)$, obtained by the least squares method, as well as of the corresponding orthogonal projection estimator $\hat{f}_{N}(t)=$ $\sum_{k=-m}^{m} \hat{c}_{k} \exp (i 2 \pi k t)$, where $N=2 m+1$, are examined and compared with those of the standard Discrete Fourier Transform.

1. Introduction. Concepts of spectral analysis of unequally spaced data considered so far are usually based on the least squares periodogram approach, i.e. fitting monochromatic harmonics to observations using the least squares method. Such harmonics have the form $a \sin (\omega t)+b \cos (\omega t)$, where $\omega>0, a, b, t \in \mathbb{R}$, or $c \exp (i \omega t), \omega \in \mathbb{R}, \omega \neq 0, c \in \mathbb{C}$, possibly with additive constant intercept term [3], [8], [9], 28], [29]. Discrete orthogonalization of the set of such base functions fitted by the least squares method was considered in [2]. Statistical aspects of the periodogram approach were investigated in particular in [4], [13, [24] and relevant algorithms together with their numerical features are examined in [11].

For equidistant observation times the periodogram values at discrete frequencies $\omega_{k}=2 \pi k / n, k=0, \pm 1, \ldots, \pm[(n-1) / 2]$, where $n$ is the number

2020 Mathematics Subject Classification: 65T40, 65T50.

Key words and phrases: least squares method, discrete Fourier transform, trigonometric polynomials, unequally spaced data.

Received 5 November 2019; revised 6 April 2020.

Published online 9 November 2020. 
of observations and $[a]$ denotes the integer part of $a \in \mathbb{R}$, can be calculated using the Discrete Fourier Transform (DFT) [5] implemented in Fast Fourier Transform numerical procedures [25]. In the present work the problem of similar discrete Fourier analysis of observations at non-equidistant times is examined. Namely, analogously to the case of standard DFT we shall determine the coefficients of a linear combination of base harmonics $\exp (i 2 \pi k t)$, $k=0, \pm 1, \ldots, \pm m$, where $m \in \mathbb{N}, t \in \mathbb{R}$, by fitting such a trigonometric polynomial to the observed data using the least squares method.

Let $y_{j}, j=1, \ldots, n$, be observations at times $t_{j} \in[0,1]$, according to the model $y_{j}=f\left(t_{j}\right)+\eta_{j}$, where $f:[0,1] \rightarrow \mathbb{C}$ is an unknown function and $\eta_{j}, j=1, \ldots, n$, are correlated complex valued random variables with zero mean value and finite variance $\sigma_{\eta}^{2}>0$. Furthermore, suppose $t_{j} \in I_{j}=[(j-1) / n, j / n), j=1, \ldots, n$, i.e. we consider an irregular design observation model. Alternatively, we can assume that $t_{j}=t_{m j}+\xi_{j}$, $j=1, \ldots, n$, represent the equidistant observation times $t_{m j}=(j-1 / 2) / n$ (midpoints of the intervals $I_{j}$ ) distorted by some bounded deterministic or random errors $\xi_{j}$ which satisfy $\left|\xi_{j}\right|<1 /(2 n), j=1, \ldots, n$; e.g., such distortions can be realizations of independent and identically distributed random variables with uniform or triangular distribution on the interval $(-1 /(2 n), 1 /(2 n))$, independent of the observation errors.

In order to approximate the unknown function $f$ we shall use the complete orthonormal system of trigonometric functions $e_{k}(t)=\exp (i 2 \pi k t)$, $t \in[0,1], k=0, \pm 1, \ldots$, from the Hilbert space $L^{2}[0,1]$, so

$$
\int_{0}^{1} e_{k}(s) \overline{e_{l}}(s) d s=\delta_{k l}, \quad k, l=0, \pm 1, \ldots,
$$

where $\delta_{k l}$ denotes the Kronecker delta. Putting

$$
e^{N}(t)=\left(e_{-m}(t), e_{-m+1}(t), \ldots, e_{m}(t)\right)^{T}
$$

for $N=2 m+1, m=0,1, \ldots$, and $t \in[0,1]$, we have

$$
\left\|e^{N}(t)\right\|^{2}=\overline{e^{N}}(t)^{T} e^{N}(t)=\sum_{k=-m}^{m}\left|e_{k}(t)\right|^{2}=2 m+1=N .
$$

Now, we can represent $f \in L^{2}[0,1]$ as

$$
f(t)=f_{N}(t)+r_{N}(t)=\sum_{k=-m}^{m} c_{k} e_{k}(t)+r_{N}(t)=e^{N}(t)^{T} c^{N}+r_{N}(t),
$$

where

$$
c^{N}=\left(c_{-m}, c_{-m+1}, \ldots, c_{m}\right)^{T}
$$


is the vector of Fourier coefficients given by

$$
c_{k}=\int_{0}^{1} f(s) \overline{e_{k}}(s) d s, \quad k=0, \pm 1, \ldots
$$

As an estimator of the vector $c^{N}$, for fixed $m$, we take the vector $\hat{c}^{N}=$ $\left(\hat{c}_{-m}, \hat{c}_{-m+1}, \ldots, \hat{c}_{m}\right)^{T}$ obtained by the least squares method, i.e.

$$
\hat{c}^{N}=\underset{a \in \mathbb{C}^{N}}{\arg \min } \sum_{j=1}^{n}\left|y_{j}-e^{N}\left(t_{j}\right)^{T} a\right|^{2},
$$

and we construct the corresponding estimator of the orthogonal projection of the function $f$ on the $N$-dimensional linear subspace $V_{m}=\operatorname{span}\left\{e_{-m}, e_{-m+1}\right.$, $\left.\ldots, e_{m}\right\}$ :

$$
\hat{f}_{N}(t)=\sum_{k=-m}^{m} \hat{c}_{k} e_{k}(t)=e^{N}(t)^{T} \hat{c}^{N} .
$$

The vector $\hat{c}^{N}$ can be obtained as a solution of the normal equations

$$
G_{n} \hat{c}^{N}=g_{n}
$$

where

$$
G_{n}=\frac{1}{n} \sum_{j=1}^{n} \overline{e^{N}}\left(t_{j}\right) e^{N}\left(t_{j}\right)^{T}, \quad g_{n}=\frac{1}{n} \sum_{j=1}^{n} y_{j} \overline{e^{N}}\left(t_{j}\right) .
$$

In view of (3) we see immediately that

$$
\hat{c}^{N}=c^{N}+G_{n}^{-1}\left(\frac{1}{n} \sum_{j=1}^{n} r_{N}\left(t_{j}\right) \overline{e^{N}}\left(t_{j}\right)\right)+G_{n}^{-1}\left(\frac{1}{n} \sum_{j=1}^{n} \eta_{j} \overline{e^{N}}\left(t_{j}\right)\right) .
$$

It should be noted that for the equidistant observation times $t_{0 j}=(j-1) / n$, $j=1, \ldots, n$, the well-known orthogonality relations [5]

$$
\frac{1}{n} \sum_{j=1}^{n} e_{k}\left(t_{0 j}\right) \overline{e_{l}}\left(t_{0 j}\right)=\frac{1}{n} \sum_{j=1}^{n} \exp (i 2 \pi(k-l)(j-1) / n)=\delta_{k l}
$$

for $k, l=0, \pm 1, \ldots, \pm[(n-1) / 2]$ imply $G_{n}=I_{N}$. Consequently, for $N=$ $2 m+1 \leq n$ the corresponding coefficients determined by the least squares method are easily computed as $\tilde{c}^{N}=\frac{1}{n} \sum_{j=1}^{n} y_{j} \overline{e^{N}}\left(t_{0 j}\right)$ or

$$
\tilde{c}_{k}=\frac{1}{n} \sum_{j=1}^{n} y_{j} \exp (-i 2 \pi k(j-1) / n) \quad \text { for } k=0, \pm 1, \ldots, \pm[(n-1) / 2]
$$

these are the standard DFT coefficients [5]. The orthogonal projection estimator corresponding to the DFT coefficients will be denoted as

$$
\tilde{f}_{N}(t)=\sum_{k=-m}^{m} \tilde{c}_{k} e_{k}(t)=e^{N}(t)^{T} \tilde{c}^{N},
$$


and for $N=2 m+1=n$ its point values $\tilde{f}_{N}\left(t_{0 j}\right)$ interpolate the observations, i.e. $\tilde{f}_{N}\left(t_{0 j}\right)=y_{j}, j=1, \ldots, n$, according to the known DFT properties [5].

We shall need the following lemmas which were proved in [20]. We repeat the proofs for completeness.

Lemma 1.1. Let $h=\left(h_{1}, \ldots, h_{n}\right)^{T} \in \mathbb{C}^{n}$. Then

$$
\frac{1}{n^{2}} \sum_{j=1}^{n} \sum_{k=1}^{n} \bar{h}_{j} h_{k} e^{N}\left(t_{j}\right)^{T} G_{n}^{-1} \overline{e^{N}}\left(t_{k}\right) \leq \frac{1}{n} \sum_{j=1}^{n}\left|h_{j}\right|^{2} .
$$

Proof. Define $a_{n}=G_{n}^{-1}\left(\frac{1}{n} \sum_{k=1}^{n} h_{k} \overline{e^{N}}\left(t_{k}\right)\right)$. The inequality

$$
\begin{aligned}
0 & \leq \frac{1}{n} \sum_{j=1}^{n}\left|h_{j}-e^{N}\left(t_{j}\right)^{T} a_{n}\right|^{2} \\
& =\frac{1}{n} \sum_{j=1}^{n}\left|h_{j}\right|^{2}-\frac{1}{n^{2}} \sum_{j=1}^{n} \sum_{k=1}^{n} \bar{h}_{j} h_{k} e^{N}\left(t_{j}\right)^{T} G_{n}^{-1} \overline{e^{N}}\left(t_{k}\right)
\end{aligned}
$$

completes the proof.

Lemma 1.2. Let $G$ be a hermitian nonnegative definite $N \times N$ matrix. Then

$$
\left\langle G^{2} a, a\right\rangle=\bar{a}^{T} G^{2} a \leq \lambda_{\max }(G) \bar{a}^{T} G a \leq\|G\| \bar{a}^{T} G a=\|G\|\langle G a, a\rangle
$$

for $a \in \mathbb{C}^{N}$, where $\lambda_{\max }(G)$ is the largest eigenvalue of $G$.

Proof. Since $G$ is hermitian and nonnegative definite, we have

$$
\begin{aligned}
\bar{a}^{T} G^{2} a & =\bar{a}^{T} G^{1 / 2} G G^{1 / 2} a \leq \lambda_{\max }(G)\left\|G^{1 / 2} a\right\|^{2} \\
& =\lambda_{\max }(G) \bar{a}^{T} G a \leq\|G\| \bar{a}^{T} G a .
\end{aligned}
$$

The outline of the work is the following. Sufficient conditions for uniqueness and numerical stability of the Fourier coefficient estimators are derived in Section 2. Section 3 is devoted to their statistical characteristics, while in Sections 4-5 finite sample and asymptotic properties of the orthogonal projection estimators constructed using the Fourier coefficient estimators are dealt with.

2. Uniqueness and stability of Fourier coefficient estimators. In order to pursue our investigations of the estimators we have to find an upper bound for $\left\|G_{n}^{-1}\right\|$, which is based on the ideas from [1]. Namely, we shall find a bound for the norm of the hermitian matrix $\Delta_{n}=I-G_{n}$ observing that for $v=\sum_{k=-m}^{m} a_{k} e_{k}$, where $a=\left(a_{-m}, a_{-m+1}, \ldots, a_{m-1}, a_{m}\right)^{T} \in \mathbb{C}^{N}$,

$$
\left\langle\Delta_{n} a, a\right\rangle=\|a\|^{2}-\left\langle G_{n} a, a\right\rangle=\|v\|_{2}^{2}-\frac{1}{n} \sum_{j=1}^{n}\left|e^{N}\left(t_{j}\right)^{T} a\right|^{2}=\|v\|_{2}^{2}-\frac{1}{n} \sum_{j=1}^{n}\left|v\left(t_{j}\right)\right|^{2} .
$$


Thus, according to the well known results on hermitian linear operators [30],

$$
\left\|\Delta_{n}\right\|=\left.\sup _{\|v\|_{2}=1}\left|\frac{1}{n} \sum_{j=1}^{n}\right| v\left(t_{j}\right)\right|^{2}-\|v\|_{2}^{2} \mid .
$$

So the next aim is to derive suffcient conditions for $\left\|\Delta_{n}\right\| \leq \alpha$, where $0<$ $\alpha<1$, which further implies that $G_{n}=I-\Delta_{n}$ is invertible and $\left\|G_{n}^{-1}\right\| \leq$ $\sum_{k=0}^{\infty} \alpha^{k}=1 /(1-\alpha)$, according to the classical results from functional analysis [23]. This can be achieved using the following lemma.

Lemma 2.1. For any trigonometric polynomial $v \in V_{m}=\operatorname{span}\left\{e_{-m}\right.$, $\left.e_{-m+1}, \ldots, e_{m-1}, e_{m}\right\}$

$$
\left.\left|\frac{1}{n} \sum_{j=1}^{n}\right| v\left(t_{j}\right)\right|^{2}-\|v\|_{2}^{2} \mid \leq \frac{8 \pi}{n} m\|v\|_{2}^{2} .
$$

Proof. Since $t_{j} \in I_{j}=[(j-1) / n, j / n), j=1, \ldots, n$, we have

$$
\begin{aligned}
\left.\left|\int_{I_{j}}\right| v(s)\right|^{2} d s-\frac{1}{n}\left|v\left(t_{j}\right)\right|^{2} \mid & =\left|\int_{I_{j}}\left(|v(s)|^{2}-\left|v\left(t_{j}\right)\right|^{2}\right) d s\right| \\
& \leq\left.\int_{I_{j}}|| v(s)\right|^{2}-\left|v\left(t_{j}\right)\right|^{2}\left|d s \leq \frac{1}{n} \int_{I_{j}} 2\right| 2 v(s) v^{\prime}(s) \mid d s \\
& =\frac{4}{n} \int_{I_{j}}\left|v(s) v^{\prime}(s)\right| d s,
\end{aligned}
$$

and by summing over all intervals $I_{j}, j=1, \ldots, n$, we have

$$
\left.\left|\int_{0}^{1}\right| v(s)\right|^{2} d s-\frac{1}{n} \sum_{j=1}^{n}\left|v\left(t_{j}\right)\right|^{2}\left|\leq \frac{4}{n} \int_{0}^{1}\right| v(s) v^{\prime}(s) \mid d s .
$$

Orthogonality of the basis functions $e_{k}, k=0, \pm 1, \ldots$, (see (1)) allows us to derive immediately the bound $\left\|v^{\prime}\right\|_{2} \leq 2 \pi m\|v\|_{2}$ (Bernstein inequality in the $L^{2}$-norm [31]). Applying the Schwarz inequality together with the Bernstein inequality proves the lemma.

Let us also recall the famous Koksma inequality for quasi Monte Carlo numerical integration [12]:

If the real valued function $g:[0,1] \rightarrow \mathbb{R}$ has bounded variation $V(g)$ on $[0,1]$ and $x_{1}, \ldots, x_{n} \in[0,1)$ are arbitrary points, then

$$
\left|\int_{0}^{1} g(s) d s-\frac{1}{n} \sum_{j=1}^{n} g\left(x_{j}\right)\right| \leq V(g) D_{n}^{*}(\mathcal{S}),
$$

where $D_{n}^{*}(\mathcal{S})$ is the star discrepancy of the point set $\mathcal{S}=\left\{x_{1}, \ldots, x_{n}\right\}$. 
The star discrepancy is defined and characterised for $0 \leq x_{1} \leq \cdots \leq$ $x_{n}<1$ as follows [12]:

$$
D_{n}^{*}(\mathcal{S})=\sup _{0<s \leq 1}\left|\frac{1}{n} \sum_{j=1}^{n} \chi_{[0, s)}\left(x_{j}\right)-s\right|=\frac{1}{2 n}+\max _{1 \leq j \leq n}\left|x_{j}-\frac{2 j-1}{2 n}\right|,
$$

where $\chi_{[0, s)}$ denotes the characteristic function of the interval $[0, s)$.

We can adapt the Koksma inequality to our case by putting $g(x)=$ $|v(x)|^{2}$ for $x \in[0,1]$ and $x_{j}=t_{j}, j=1, \ldots, n$, which results in

$$
\left.\left|\int_{0}^{1}\right| v(s)\right|^{2} d s-\frac{1}{n} \sum_{j=1}^{n}\left|v\left(t_{j}\right)\right|^{2} \mid \leq\left(V\left(\Re[v]^{2}\right)+V\left(\Im[v]^{2}\right)\right) D_{n}^{*}(S),
$$

where

$$
D_{n}^{*}(\mathcal{S})=\frac{1}{2 n}+\max _{1 \leq j \leq n}\left|t_{j}-\frac{2 j-1}{2 n}\right|=\frac{1}{2 n}+\max _{1 \leq j \leq n}\left|t_{j}-t_{m j}\right|,
$$

and the sum of variations can be easily estimated using the Schwarz inequality:

$$
V\left(\Re[v]^{2}\right)+V\left(\Im[v]^{2}\right) \leq 2 \int_{0}^{1}\left|2 v(s) v^{\prime}(s)\right| d s \leq 4\|v\|_{2}\left\|v^{\prime}\right\|_{2} \leq 8 \pi m\|v\|_{2}^{2} .
$$

Hence, a lemma similar to 2.1 can be proved in the more general case of $t_{j}, t_{m j} \in J_{j}$, where the intervals $J_{j}, j=1, \ldots, n$, form a partition of $[0,1]$ (i.e. are disjoint and $\bigcup_{j=1}^{n} J_{j}=[0,1]$ ), and $\max _{1 \leq j \leq n}\left|J_{j}\right| \leq C / n$ for $C>0$.

Now, it is easy to see from (7) and Lemma 2.1 that $\left\|\Delta_{n}\right\| \leq \alpha$ for $m$ such that $8 \pi m / n \leq \alpha$, which means that invertibility of $G_{n}$ together with the inequality $\left\|G_{n}^{-1}\right\| \leq 1 /(1-\alpha)$ is ensured for $0<\alpha<1$ and

$$
m \leq n \alpha /(8 \pi)<n /(8 \pi) \text {. }
$$

Since $\alpha_{0}=[n /(8 \pi)] 8 \pi / n<1$, the above inequality shows that it is possible to compute uniquely $N=2 m+1$ coefficients, where $m \leq[n /(8 \pi)]$.

Moreover, if $H_{n}$ is the $n \times N$ matrix with elements $h_{j k}=e_{k-m-1}\left(t_{j}\right) / n$, $j=1, \ldots, n, k=1, \ldots, 2 m+1=N$, then obviously $G_{n}=n H_{n}^{*} H_{n}, g_{n}=$ $H_{n}^{*} y$, where $M^{*}$ denotes the hermitian transpose of any matrix $M$ with complex entries and $y=\left(y_{1}, \ldots, y_{n}\right)^{T}$ is the vector of observations. So for any vector $a \in \mathbb{C}^{N}$,

$$
\left\langle G_{n} a, a\right\rangle=n\left\langle H_{n}^{*} H_{n} a, a\right\rangle=n\left\langle H_{n} a, H_{n} a\right\rangle=n\left\|H_{n} a\right\|^{2},
$$

which yields $\left\|H_{n}\right\|=\left\|H_{n}^{*}\right\|=\left(\left\|G_{n}\right\| / n\right)^{1 / 2}$. Therefore, if condition (8) is satisfied, then also $\left\|G_{n}\right\|=\left\|I-\Delta_{n}\right\| \leq 1+\alpha<2$, and in consequence by (4),

$$
\left\|\hat{c}^{N}\right\| \leq\left\|G_{n}^{-1}\right\|\left\|g_{n}\right\| \leq \frac{1}{1-\alpha}\left\|H_{n}^{*}\right\|\|y\|<\frac{2^{1 / 2}}{n^{1 / 2}(1-\alpha)}\|y\| .
$$

This ensures stability of the estimates $\hat{c}^{N}$. 
In the DFT case the vectors $\left(e_{k}\left(t_{01}\right), e_{k}\left(t_{02}\right), \ldots, e_{k}\left(t_{0 n}\right)\right)^{T}, k=0, \pm 1$, $\ldots, \pm[(n-1) / 2)]$, are orthogonal by (6), while for other observation times the vectors $\left(e_{k}\left(t_{1}\right), \ldots, e_{k}\left(t_{n}\right)\right)^{T}, k=0, \pm 1, \ldots, \pm[n /(8 \pi)]$, (i.e. the columns of the matrix $n H_{n}$ ) are linearly independent, which follows from the invertibility of $G_{n}=n H_{n}^{*} H_{n}$. Hence, using Gram-Schmidt orthonormalization in $\mathbb{C}^{n}$, we can get the standard QR decomposition $n H_{n}=Q_{n} R_{n}$, where $Q_{n}$ is a unitary matrix and $R_{n}$ is a nonsingular upper triangular matrix. Further the equality $\left\|y-n H_{n} \hat{c}^{N}\right\|=\left\|y-Q_{n} R_{n} \hat{c}^{N}\right\|$ implies that the least squares coefficient estimators corresponding to the orthonormal basis are obtained by the linear transformation $R_{n} \hat{c}^{N}$. A similar orthogonalization concept was proposed by Ferraz-Mello [2] who used only three basis functions $1, \sin (\omega t)$, $\cos (\omega t)$, where $\omega>0$, to approximate real valued observation data.

3. Statistical characteristics of the Fourier coefficient estimators. Let us now estimate the squared bias $\left\|c^{N}-E_{\eta} \hat{c}^{N}\right\|^{2}$ and variance $E_{\eta}\left\|\hat{c}^{N}-E_{\eta} \hat{c}^{N}\right\|^{2}$ of the estimators $\hat{c}^{N}$, which is the subject of the two lemmas below.

Lemma 3.1. If $m \leq[n \alpha /(8 \pi)]$, where $0<\alpha \leq[n /(8 \pi)] 8 \pi / n$, and $N=$ $2 m+1$, then

$$
E_{\eta}\left\|\hat{c}^{N}-E_{\eta} \hat{c}^{N}\right\|^{2} \leq \frac{N}{n^{2}(1-\alpha)^{2}} \sum_{j=1}^{n} \sum_{k=1}^{n}\left|E_{\eta} \eta_{j} \overline{\eta_{k}}\right|
$$

for correlated observation errors, and

for uncorrelated errors.

$$
\frac{N}{2 n} \sigma_{\eta}^{2}<E_{\eta}\left\|\hat{c}^{N}-E_{\eta} \hat{c}^{N}\right\|^{2} \leq \frac{N}{n(1-\alpha)} \sigma_{\eta}^{2}
$$
then

In the DFT case, where $t_{0 j}=(j-1) / n, j=1, \ldots, n$, if $m \leq[(n-1) / 2]$,

$$
E_{\eta}\left\|\tilde{c}^{N}-E_{\eta} \tilde{c}^{N}\right\|^{2} \leq \frac{N}{n^{2}} \sum_{j=1}^{n} \sum_{k=1}^{n}\left|E_{\eta} \eta_{j} \overline{\eta_{k}}\right|
$$

for correlated observation errors, and

for uncorrelated errors.

$$
E_{\eta}\left\|\tilde{c}^{N}-E_{\eta} \tilde{c}^{N}\right\|^{2}=\frac{N}{n} \sigma_{\eta}^{2}
$$

Proof. In view of decomposition (5) we obtain immediately

$$
\begin{aligned}
E_{\eta}\left\|\hat{c}^{N}-E_{\eta} \hat{c}^{N}\right\|^{2} & =E_{\eta}\left\|G_{n}^{-1}\left(\frac{1}{n} \sum_{j=1}^{n} \eta_{j} \overline{e^{N}}\left(t_{j}\right)\right)\right\|^{2} \\
& =\frac{1}{n^{2}} \sum_{j=1}^{n} \sum_{k=1}^{n} E_{\eta} \eta_{j} \overline{\eta_{k}}\left\langle G_{n}^{-1} \overline{e^{N}}\left(t_{j}\right), G_{n}^{-1} \overline{e^{N}}\left(t_{k}\right)\right\rangle
\end{aligned}
$$




$$
\begin{aligned}
& \leq \frac{1}{n^{2}} \sum_{j=1}^{n} \sum_{k=1}^{n}\left|E_{\eta} \eta_{j} \overline{\eta_{k}}\right|\left\|G_{n}^{-1}\right\|^{2}\left\|e^{N}\left(t_{j}\right)\right\|\left\|e^{N}\left(t_{k}\right)\right\| \\
& \leq \frac{N}{n^{2}(1-\alpha)^{2}} \sum_{j=1}^{n} \sum_{k=1}^{n}\left|E_{\eta} \eta_{j} \overline{\eta_{k}}\right|,
\end{aligned}
$$

where we have used the Schwarz inequality and the bound $\left\|G_{n}^{-1}\right\| \leq(1-\alpha)^{-1}$ together with (2), so the proof for correlated errors is complete.

For uncorrelated errors we obtain analogously

$$
\begin{aligned}
E_{\eta}\left\|\hat{c}^{N}-E_{\eta} \hat{c}^{N}\right\|^{2} & =\frac{\sigma_{\eta}^{2}}{n^{2}} \sum_{j=1}^{n} \operatorname{tr}\left(e^{N}\left(t_{j}\right)^{T} G_{n}^{-1} G_{n}^{-1} \overline{e^{N}}\left(t_{j}\right)\right) \\
& =\frac{\sigma_{\eta}^{2}}{n} \operatorname{tr}\left(\frac{1}{n} \sum_{j=1}^{n} \overline{e^{N}}\left(t_{j}\right) e^{N}\left(t_{j}\right)^{T} G_{n}^{-1} G_{n}^{-1}\right) \\
& =\frac{\sigma_{\eta}^{2}}{n} \operatorname{tr}\left(G_{n} G_{n}^{-1} G_{n}^{-1}\right)=\frac{\sigma_{\eta}^{2}}{n} \operatorname{tr}\left(G_{n}^{-1}\right),
\end{aligned}
$$

and further

$$
\frac{N}{2 n} \sigma_{\eta}^{2}<\frac{\sigma_{\eta}^{2}}{n} \operatorname{tr}\left(G_{n}^{-1}\right) \leq \frac{N}{n(1-\alpha)} \sigma_{\eta}^{2},
$$

since $\lambda_{\max }\left(G_{n}^{-1}\right) \leq\left\|G_{n}^{-1}\right\| \leq(1-\alpha)^{-1}$ and $\lambda_{\min }\left(G_{n}^{-1}\right)=\lambda_{\max }\left(G_{n}\right)^{-1} \geq$ $\left\|G_{n}\right\|^{-1} \geq(1+\alpha)^{-1}>1 / 2$ (as remarked in Section 2 , the condition of the lemma ensures that $\left\|G_{n}^{-1}\right\| \leq(1-\alpha)^{-1}$ as well as $\left.\left\|G_{n}\right\| \leq 1+\alpha<2\right)$, and the lemma is proved.

LEMMA 3.2. If $m \leq[n \alpha /(8 \pi)]$, where $0<\alpha \leq[n /(8 \pi)] 8 \pi / n$, and $r_{N}=$ $f-f_{N}$, where $N=2 m+1$, then

$$
\left\|c^{N}-E_{\eta} \hat{c}^{N}\right\|^{2} \leq \frac{1}{n(1-\alpha)} \sum_{j=1}^{n}\left|r_{N}\left(t_{j}\right)\right|^{2} .
$$

In the DFT case, where $t_{0 j}=(j-1) / n, j=1, \ldots, n$, if $m \leq[(n-1) / 2]$, then

$$
\left\|c^{N}-E_{\eta} \tilde{c}^{N}\right\|^{2} \leq \frac{1}{n} \sum_{j=1}^{n}\left|r_{N}\left(t_{0 j}\right)\right|^{2}
$$

Proof. Using again the decomposition (5) and putting

$$
a_{n}=\frac{1}{n} \sum_{k=1}^{n} r_{N}\left(t_{k}\right) \overline{e^{N}}\left(t_{k}\right)
$$


we can write, taking into account Lemmas 1.1 and 1.2 ,

$$
\begin{aligned}
\left\|c^{N}-E_{\eta} \hat{c}^{N}\right\|^{2} & =\left\|G_{n}^{-1} a_{n}\right\|^{2}=\left\langle G_{n}^{-1} G_{n}^{-1} a_{n}, a_{n}\right\rangle \leq(1-\alpha)^{-1}\left\langle G_{n}^{-1} a_{n}, a_{n}\right\rangle \\
& =\frac{1}{n^{2}(1-\alpha)} \sum_{j=1}^{n} \sum_{k=1}^{n} \overline{r_{N}}\left(t_{j}\right) r_{N}\left(t_{k}\right) e^{N}\left(t_{j}\right)^{T} G_{n}^{-1} \overline{e^{N}}\left(t_{k}\right) \\
& \leq \frac{1}{n(1-\alpha)} \sum_{j=1}^{n}\left|r_{N}\left(t_{j}\right)\right|^{2},
\end{aligned}
$$

which proves our assertion.

In the DFT case the orthogonality relations (6) ensure that for $m \leq$ $[(n-1) / 2]$,

$\tilde{s}_{N}=\frac{1}{n} \sum_{j=1}^{n}\left|y_{j}-e^{N}\left(t_{0 j}\right)^{T} \tilde{c}^{N}\right|^{2}=\frac{1}{n} \sum_{j=1}^{n}\left|y_{j}\right|^{2}-\sum_{k=-m}^{m}\left|\tilde{c}_{k}\right|^{2}=\frac{1}{n}\|y\|^{2}-\left\|\tilde{c}^{N}\right\|^{2}$,

and so $\left\|\tilde{c}^{N}\right\|^{2} \leq\|y\|^{2} / n$ (equality holds for $N=2 m+1=n$ ). For $t_{j} \in$ $[(j-1) / n, j / n), j=1, \ldots, n$, the well known properties of the orthogonal projection related to the estimator $\hat{c}^{N}$ yield

$$
\begin{aligned}
\hat{s}_{N} & =\frac{1}{n} \sum_{j=1}^{n}\left|y_{j}-e^{N}\left(t_{j}\right)^{T} c^{N}\right|^{2}=\frac{1}{n}\left\|y-n H_{n} \hat{c}^{N}\right\|^{2} \\
& =\frac{1}{n}\left\langle y-n H_{n} \hat{c}^{N}, y-n H_{n} \hat{c}^{N}\right\rangle=\frac{1}{n}\left\langle y-n H_{n} \hat{c}^{N}, y\right\rangle \\
& =\frac{1}{n}\|y\|^{2}-n \frac{1}{n}\left\langle H_{n} \hat{c}^{N}, y\right\rangle=\frac{1}{n}\|y\|^{2}-\left\langle G_{n}^{-1} H_{n}^{*} y, H_{n}^{*} y\right\rangle,
\end{aligned}
$$

where we have used the equality $\hat{c}_{N}=G_{n}^{-1} g_{n}=G_{n}^{-1} H_{n}^{*} y$. Obviously $H_{n}^{*} y=$ $\left(\hat{d}_{-m}, \hat{d}_{-m+1}, \ldots, \hat{d}_{m}\right)^{T}$, where $d_{k}=\frac{1}{n} \sum_{j=1}^{n} y_{j} \overline{e_{k}}\left(t_{j}\right), k=0, \pm 1, \ldots, \pm m$, $m \leq[n /(8 \pi)]$. One can see immediately the similarity of the formula for $\tilde{s}_{N}$ and the one for $\hat{s}_{N}$.

As remarked in the proof of Lemma 3.1, for $m \leq[n \alpha /(8 \pi)]$, where $0<$ $\alpha \leq[n /(8 \pi)] 8 \pi / n$, we know that $\lambda_{\max }\left(G_{n}^{-1}\right) \leq\left\|G_{n}^{-1}\right\| \leq(1-\alpha)^{-1}$ and $\lambda_{\min }\left(G_{n}^{-1}\right)=\lambda_{\max }\left(G_{n}\right)^{-1} \geq\left\|G_{n}\right\|^{-1} \geq(1+\alpha)^{-1}>1 / 2$, so we easily obtain the following lower and upper bounds on $\hat{s}_{N}$ :

$$
\frac{1}{n}\|y\|^{2}-\frac{1}{1-\alpha} \bar{y}^{T} H_{n} H_{n}^{*} y \leq \hat{s}_{N} \leq \frac{1}{n}\|y\|^{2}-\frac{1}{2} \bar{y}^{T} H_{n} H_{n}^{*} y,
$$

or otherwise

$$
\frac{1}{n}\|y\|^{2}-\frac{1}{1-\alpha} \sum_{k=-m}^{m}\left|\hat{d}_{k}\right|^{2} \leq \hat{s}_{N} \leq \frac{1}{n}\|y\|^{2}-\frac{1}{2} \sum_{k=-m}^{m}\left|\hat{d}_{k}\right|^{2} .
$$

Since the vector $\hat{c}^{N}$ is obtained by a linear transformation of the vector of observations $y$, in the case of gaussian observation errors the coefficient 
estimators also have gaussian distribution. This fact enables construction of confidence intervals with specified significance level for such coefficients [22].

4. Properties of the projection estimators. Let us observe that for $m \leq[n \alpha /(8 \pi)]$, where $0<\alpha \leq[n /(8 \pi)] 8 \pi / n$, the following bound can be deduced from (9) using the Schwarz inequality together with (2):

$$
\begin{aligned}
\left|\hat{f}_{N}(t)\right| & \leq N^{1 / 2}\left\|\hat{c}^{N}\right\| \leq \frac{(2 m+1)^{1 / 2} 2^{1 / 2}}{(1-\alpha) n^{1 / 2}}\|y\| \\
& \leq\left[\frac{1}{2^{1 / 2} \pi^{1 / 2}(1-\alpha)}+\frac{2^{1 / 2}}{(1-\alpha) n^{1 / 2}}\right]\|y\|
\end{aligned}
$$

for $t \in[0,1]$, i.e. the estimator represents a continuous linear operator $\hat{f}_{N}$ : $\mathbb{C}^{n} \rightarrow C[0,1]$. Similarly in the DFT case the inequality $\left\|\tilde{c}^{N}\right\|^{2} \leq\|y\|^{2} / n$ valid for $m \leq[(n-1) / 2]$ yields

$$
\left|\tilde{f}_{N}(t)\right| \leq N^{1 / 2}\left\|\tilde{c}^{N}\right\| \leq \frac{(2 m+1)^{1 / 2}}{n^{1 / 2}}\|y\| \leq\|y\|
$$

for $t \in[0,1]$.

One can also examine the average sample error

$$
R\left(\hat{f}_{N}\right)=E_{\eta} \frac{1}{n} \sum_{j=1}^{n}\left|f\left(t_{j}\right)-\hat{f}_{N}\left(t_{j}\right)\right|^{2},
$$

which is an approximation of the Integrated Mean Square Error (IMSE):

$$
R\left(\hat{f}_{N}\right)=\frac{1}{n} \sum_{j=1}^{n} E_{\eta}\left|f\left(t_{j}\right)-\hat{f}_{N}\left(t_{j}\right)\right|^{2} \cong E_{\eta}\left\|f-\hat{f}_{N}\right\|_{2}^{2},
$$

since $t_{j} \in I_{j}=[(j-1) / n, j / n), j=1,2, \ldots, n$. The standard variance plus squared bias decomposition of $R\left(\hat{f}_{N}\right)$ yields

$$
\begin{aligned}
E_{\eta} \frac{1}{n} \sum_{j=1}^{n} & \left|f\left(t_{j}\right)-\hat{f}_{N}\left(t_{j}\right)\right|^{2} \\
& =\frac{1}{n} \sum_{j=1}^{n}\left|f\left(t_{j}\right)-E_{\eta} \hat{f}_{N}\left(t_{j}\right)\right|^{2}+\frac{1}{n} \sum_{j=1}^{n} E_{\eta}\left|\hat{f}_{N}\left(t_{j}\right)-E_{\eta} \hat{f}_{N}\left(t_{j}\right)\right|^{2} \\
& \leq \frac{1}{n} \sum_{j=1}^{n} E_{\eta}\left|e^{N}\left(t_{j}\right)^{T}\left(\hat{c}^{N}-E_{\eta} \hat{c}^{N}\right)\right|^{2}+\frac{1}{n} \sum_{j=1}^{n}\left|f\left(t_{j}\right)-v\left(t_{j}\right)\right|^{2}
\end{aligned}
$$

for $v \in V_{m}=\operatorname{span}\left\{e_{-m}, e_{-m+1}, \ldots, e_{m}\right\}$, since the least squares estimation principle implies that for such a trigonometric polynomial,

$$
\frac{1}{n} \sum_{j=1}^{n}\left|f\left(t_{j}\right)-E_{\eta} \hat{f}_{N}\left(t_{j}\right)\right|^{2} \leq \frac{1}{n} \sum_{j=1}^{n}\left|f\left(t_{j}\right)-v\left(t_{j}\right)\right|^{2} .
$$


It follows that for noiseless observations of $f \in V_{m}$, and $N=2 m+1$, the projection estimator $\hat{f}_{N}$ interpolates the function values $f\left(t_{j}\right), j=1, \ldots, n$, if $m \leq[n /(8 \pi)]$.

The sum of the variance terms is computed in the following lemma.

Lemma 4.1. If $m \leq[n \alpha /(8 \pi)]$, where $0<\alpha \leq[n /(8 \pi)] 8 \pi / n$, and $N=$ $2 m+1$, then

$$
\frac{1}{n} \sum_{l=1}^{n} E_{\eta}\left|e^{N}\left(t_{l}\right)^{T}\left(\hat{c}^{N}-E_{\eta} \hat{c}^{N}\right)\right|^{2} \leq \frac{N}{n^{2}(1-\alpha)} \sum_{j=1}^{n} \sum_{k=1}^{n}\left|E_{\eta} \eta_{j} \overline{\eta_{k}}\right|
$$

for correlated observation errors, and

$$
\frac{1}{n} \sum_{l=1}^{n} E_{\eta}\left|e^{N}\left(t_{l}\right)^{T}\left(\hat{c}^{N}-E_{\eta} \hat{c}^{N}\right)\right|^{2}=\frac{N}{n} \sigma_{\eta}^{2}
$$

for uncorrelated errors.

In the DFT case, where $t_{0 l}=(l-1) / n, l=1, \ldots, n$, if $m \leq[(n-1) / 2]$, then

$$
\frac{1}{n} \sum_{l=1}^{n} E_{\eta}\left|e^{N}\left(t_{0 l}\right)^{T}\left(\tilde{c}^{N}-E_{\eta} \tilde{c}^{N}\right)\right|^{2} \leq \frac{N}{n^{2}} \sum_{j=1}^{n} \sum_{k=1}^{n}\left|E_{\eta} \eta_{j} \overline{\eta_{k}}\right|
$$

for correlated observation errors, and

$$
\frac{1}{n} \sum_{l=1}^{n} E_{\eta}\left|e^{N}\left(t_{0 l}\right)^{T}\left(\tilde{c}^{N}-E_{\eta} \tilde{c}^{N}\right)\right|^{2}=\frac{N}{n} \sigma_{\eta}^{2}
$$

for uncorrelated errors.

Proof. As in the earlier proofs, using the decomposition (5) we can write

$$
\begin{gathered}
\frac{1}{n} \sum_{l=1}^{n} E_{\eta}\left|e^{N}\left(t_{l}\right)^{T}\left(\hat{c}^{N}-E_{\eta} \hat{c}^{N}\right)\right|^{2}=\frac{1}{n} \sum_{l=1}^{n} E_{\eta}\left|e^{N}\left(t_{l}\right)^{T} G_{n}^{-1}\left(\frac{1}{n} \sum_{j=1}^{n} \eta_{j} \overline{e^{N}}\left(t_{j}\right)\right)\right|^{2} \\
=\frac{1}{n^{3}} \sum_{l=1}^{n} \sum_{j=1}^{n} \sum_{k=1}^{n} E_{\eta} \eta_{j} \overline{\eta_{k}} e^{N}\left(t_{k}\right)^{T} G_{n}^{-1} \overline{e^{N}}\left(t_{l}\right) e^{N}\left(t_{l}\right)^{T} G_{n}^{-1} \overline{e^{N}}\left(t_{j}\right) \\
=\frac{1}{n^{2}} \sum_{j=1}^{n} \sum_{k=1}^{n} E_{\eta} \eta_{j} \overline{\eta_{k}} e^{N}\left(t_{k}\right)^{T} G_{n}^{-1}\left(\frac{1}{n} \sum_{l=1}^{n} \overline{e^{N}}\left(t_{l}\right) e^{N}\left(t_{l}\right)^{T}\right) G_{n}^{-1} \overline{e^{N}}\left(t_{j}\right) \\
=\frac{1}{n^{2}} \sum_{j=1}^{n} \sum_{k=1}^{n} E_{\eta} \eta_{j} \overline{\eta_{k}} e^{N}\left(t_{k}\right)^{T} G_{n}^{-1} \overline{e^{N}}\left(t_{j}\right) \\
\leq \frac{1}{n^{2}(1-\alpha)} \sum_{j=1}^{n} \sum_{k=1}^{n}\left|E_{\eta} \eta_{j} \overline{\eta_{k}}\right|\left\|e^{N}\left(t_{k}\right)\right\|\left\|e^{N}\left(t_{j}\right)\right\|,
\end{gathered}
$$


where we have used the bound $\left\|G_{n}^{-1}\right\| \leq(1-\alpha)^{-1}$, so the first assertion is proved in view of (2). For uncorrelated observation errors we analogously obtain

$$
\begin{gathered}
\frac{1}{n} \sum_{l=1}^{n} E_{\eta}\left|e^{N}\left(t_{l}\right)^{T}\left(\hat{c}^{N}-E_{\eta} \hat{c}^{N}\right)\right|^{2}=\frac{\sigma_{\eta}^{2}}{n^{2}} \sum_{k=1}^{n} \operatorname{tr}\left(e^{N}\left(t_{k}\right)^{T} G_{n}^{-1} \overline{e^{N}}\left(t_{k}\right)\right) \\
=\frac{\sigma_{\eta}^{2}}{n} \operatorname{tr}\left(\frac{1}{n} \sum_{k=1}^{n} \overline{e^{N}}\left(t_{k}\right) e^{N}\left(t_{k}\right)^{T} G_{n}^{-1}\right)=\frac{\sigma_{\eta}^{2}}{n} \operatorname{tr}\left(G_{n} G_{n}^{-1}\right)=\frac{N}{n} \sigma_{\eta}^{2},
\end{gathered}
$$

which is our second assertion.

Hence, in the case of uncorrelated observation errors we have, for $m \leq$ $[n /(8 \pi)]$ and $v \in V_{m}$,

$$
\frac{N}{n} \sigma_{\eta}^{2} \leq R\left(\hat{f}_{N}\right) \leq \frac{1}{n} \sum_{j=1}^{n}\left|f\left(t_{j}\right)-v\left(t_{j}\right)\right|^{2}+\frac{N}{n} \sigma_{\eta}^{2} .
$$

Furthermore, in view of the upper bound on the average sample error $R\left(\hat{f}_{N}\right)$ and the classical results on uniform trigonometric approximation of continuous periodic functions [31], we can easily obtain conditions for convergence of this error to zero for such functions. Namely, we can formulate the following theorem.

THEOREM 4.1. If the observation errors are uncorrelated and the sequence of natural numbers $m(n), n=1,2 \ldots$, satisfies

$$
m(n) \leq[n /(8 \pi)], \quad \lim _{n \rightarrow \infty} m(n)=\infty, \quad \lim _{n \rightarrow \infty} m(n) / n=0,
$$

and $N(n)=2 m(n)+1$, then the projection estimator $\hat{f}_{N(n)}$ of the regression function $f \in C[0,1]$ such that $f(0)=f(1)$ is consistent in the sense of the average sample error, i.e.

$$
\lim _{n \rightarrow \infty} R\left(\hat{f}_{N(n)}\right)=0
$$

A similar result for a wider function class can be obtained for observation times $t_{j}=t_{m j}+\xi_{j}, j=1, \ldots, n$, where the equidistant times $t_{m j}=$ $(j-1 / 2) / n$ are distorted by some random errors $\xi_{j}$ which are independent and have identical distribution supported on $(-1 /(2 n), 1 /(2 n))$, and are independent of the observation errors $\eta_{j}, j=1, \ldots, n$.

Observe first that for any probability density function $\varrho \in L^{1}(-1,1)$ the function $\varrho_{n}(s)=2 n \varrho(2 n s)$ is a probability density on $(-1 /(2 n), 1 /(2 n)), n=$ $1,2, \ldots$, and if $\varrho$ is bounded, i.e. $0 \leq \varrho \leq M<\infty$, then $0 \leq \varrho_{n} \leq 2 n M$. Hence, assume that the distribution of the random distortions $\xi_{j}, j=$ $1, \ldots, n$, is represented by a bounded probability density $\varrho \in L^{1}(-1,1)$. 
Now, putting $v=f_{N} \in V_{m}$ for $N=2 m+1$ and $t_{j}=t_{m j}+\xi_{j}, j=1, \ldots, n$, in (10) yields

$$
\frac{N}{n} \sigma_{\eta}^{2} \leq E_{\xi} R\left(\hat{f}_{N}\right) \leq \frac{1}{n} \sum_{j=1}^{n} E_{\xi}\left|f\left(t_{m j}+\xi_{j}\right)-f_{N}\left(t_{m j}+\xi_{j}\right)\right|^{2}+\frac{N}{n} \sigma_{\eta}^{2},
$$

which under our assumptions on distortions gives

$$
\begin{aligned}
\frac{N}{n} \sigma_{\eta}^{2} & \leq E_{\xi} R\left(\hat{f}_{N}\right)=E_{\xi} E_{\eta} \frac{1}{n} \sum_{j=1}^{n}\left|f\left(t_{m j}+\xi_{j}\right)-\hat{f}_{N}\left(t_{m j}+\xi_{j}\right)\right|^{2} \\
& \leq \frac{1}{n} \sum_{j=1}^{n} \int_{-1 /(2 n)}^{1 /(2 n)}\left|f\left(t_{m j}+s\right)-f_{N}\left(t_{m j}+s\right)\right|^{2} 2 n \varrho(2 n s) d s+\frac{N}{n} \sigma_{\eta}^{2} \\
& \leq 2 M \sum_{j=1}^{n} \int_{(j-1) / n}^{j / n}\left|f(u)-f_{N}(u)\right|^{2} d u+\frac{N}{n} \sigma_{\eta}^{2}=2 M\left\|f-f_{N}\right\|_{2}^{2}+\frac{N}{n} \sigma_{\eta}^{2} .
\end{aligned}
$$

Hence, we can formulate the following theorem.

THEOREM 4.2. If the observation errors are uncorrelated, the density of distortions distribution $\varrho \in L^{1}(-1,1)$ is bounded by $M$, and $m(n) \leq$ $[n /(8 \pi)]$, then for $N(n)=2 m(n)+1$ and $f \in L^{2}[0,1]$ the average sample error of the projection estimator $\hat{f}_{N(n)}$ satisfies

$$
\begin{aligned}
\frac{N(n)}{n} \sigma_{\eta}^{2} & \leq E_{\xi} E_{\eta} \frac{1}{n} \sum_{j=1}^{n}\left|f\left((j-1 / 2) / n+\xi_{j}\right)-\hat{f}_{N(n)}\left((j-1 / 2) / n+\xi_{j}\right)\right|^{2} \\
& \leq 2 M\left\|f-f_{N(n)}\right\|_{2}^{2}+\frac{N(n)}{n} \sigma_{\eta}^{2} .
\end{aligned}
$$

Of course for any $f \in L^{2}[0,1]$ we have $\left\|f-f_{N}\right\|_{2}^{2} \rightarrow 0$ as $N \rightarrow \infty$. Moreover, in the case of observation times involving distortions, the lemmas of the previous section enable proving another theorem on convergence of the Integrated Mean Square Error of the projection estimator for $f \in L^{2}[0,1]$.

THEOREM 4.3. If the observation errors are uncorrelated, the density $\varrho \in L^{1}(-1,1)$ of distortions is bounded by $M$, and $m(n) \leq[n /(16 \pi)]$, then for $N(n)=2 m(n)+1$ and $f \in L^{2}[0,1]$ the Integrated Mean Square Error of the projection estimator $\hat{f}_{N(n)}$ satisfies

$$
\frac{N(n)}{2 n} \sigma_{\eta}^{2}<E_{\xi} E_{\eta}\left\|f-\hat{f}_{N(n)}\right\|_{2}^{2} \leq(1+4 M)\left\|f-f_{N(n)}\right\|_{2}^{2}+\frac{2 N(n)}{n} \sigma_{\eta}^{2} .
$$

Proof. The condition $m(n) \leq[n /(16 \pi)]$ corresponds to choosing $\alpha=$ $1 / 2$ in (8), so it ensures existence of the projection estimator $\hat{f}_{N(n)}$, and taking into account orthonormality of the trigonometric system used in its 
construction (see (1)) we can write

$$
\begin{aligned}
E_{\eta} \| f & -\hat{f}_{N(n)}\left\|_{2}^{2}=E_{\eta}\right\| f-f_{N(n)}+f_{N(n)}-\hat{f}_{N(n)} \|_{2}^{2} \\
& =\left\|f-f_{N(n)}\right\|_{2}^{2}+E_{\eta}\left\|f_{N(n)}-\hat{f}_{N(n)}\right\|_{2}^{2} \\
& =\left\|f-f_{N(n)}\right\|_{2}^{2}+E_{\eta}\left\|c^{N(n)}-\hat{c}^{N(n)}\right\|^{2} \\
& =\left\|f-f_{N(n)}\right\|_{2}^{2}+\left\|c^{N(n)}-E_{\eta} \hat{c}^{N(n)}\right\|^{2}+E_{\eta}\left\|c^{N(n)}-E_{\eta} \hat{c}^{N(n)}\right\|^{2} .
\end{aligned}
$$

For $\alpha=1 / 2$ Lemmas 3.1 and 3.2 yield further

$$
\begin{aligned}
\frac{N(n)}{2 n} \sigma_{\eta}^{2} & <E_{\eta}\left\|f-\hat{f}_{N(n)}\right\|_{2}^{2} \\
& \leq\left\|f-f_{N(n)}\right\|_{2}^{2}+\frac{2 N(n)}{n} \sigma_{\eta}^{2}+\frac{2}{n} \sum_{j=1}^{n}\left|r_{N(n)}\left(t_{j}\right)\right|^{2},
\end{aligned}
$$

where $r_{N}=f-f_{N}, N=1,2, \ldots$, and $t_{j}=t_{m j}+\xi_{j}, j=1, \ldots, n$, so $E_{\xi} E_{\eta}\left\|f-\hat{f}_{N(n)}\right\|_{2}^{2} \leq\left\|f-f_{N(n)}\right\|_{2}^{2}+\frac{2 N(n)}{n} \sigma_{\eta}^{2}+\frac{2}{n} \sum_{j=1}^{n} E_{\xi}\left|r_{N(n)}\left(t_{m j}+\xi_{j}\right)\right|^{2}$.

The last term has the following upper bound:

$$
\begin{aligned}
\frac{2}{n} \sum_{j=1}^{n} E_{\xi}\left|r_{N(n)}\left(t_{m j}+\xi_{j}\right)\right|^{2} & =\frac{2}{n} \sum_{j=1}^{n} \int_{-1 /(2 n)}^{1 /(2 n)}\left|r_{N(n)}\left(t_{m j}+s\right)\right|^{2} 2 n \varrho(2 n s) d s \\
& \leq 4 M \sum_{j=1}^{n} \int_{(j-1) / n}^{j / n}\left|r_{N(n)}(u)\right|^{2} d u=4 M\left\|r_{N(n)}\right\|_{2}^{2} .
\end{aligned}
$$

Since $r_{N}=f-f_{N}$, we finally obtain

$$
\frac{N(n)}{2 n} \sigma_{\eta}^{2}<E_{\xi} E_{\eta}\left\|f-\hat{f}_{N(n)}\right\|_{2}^{2} \leq(1+4 M)\left\|f-f_{N(n)}\right\|_{2}^{2}+\frac{2 N(n)}{n} \sigma_{\eta}^{2} .
$$

5. Extension to the case of stationary observation errors with short or long range dependence. The results of Sections 3 and 4 can be readily adapted to the case of stationary correlated observation errors $\eta_{j}$, $j=1, \ldots, n$, with short and long range dependence. In such a case we assume that $E_{\eta} \eta_{j}=0, E_{\eta}\left|\eta_{j}\right|^{2}=\sigma_{\eta}^{2}, j=1,2, \ldots$, and $E_{\eta} \eta_{j} \overline{\eta_{j+l}}=r(l), l=1,2, \ldots$, so

$$
\frac{1}{n^{2}} \sum_{j=1}^{n} \sum_{k=1}^{n}\left|E_{\eta} \eta_{j} \overline{\eta_{k}}\right|=\frac{\sigma_{\eta}^{2}}{n}+\frac{2}{n^{2}} \sum_{l=1}^{n-1}(n-l)|r(l)| \leq \frac{\sigma_{\eta}^{2}}{n}+\frac{2}{n} \sum_{l=1}^{\infty}|r(l)| .
$$

For short range dependent observation errors we have $\sum_{l=1}^{\infty}|r(l)|<\infty$, and then the last inequality can be merged with the relevant estimates for correlated observation errors in Lemmas 3.1 and 4.1. 
The class of long range dependent observation errors is characterized by $\sum_{l=1}^{\infty}|r(l)|=\infty$. Assume that $|r(l)|=C / l^{\gamma}, l=1,2, \ldots$, where $C>0$ and $0<\gamma<1$. Then the sum on the left hand side in (11), which is due to dependence of these errors, satisfies the known inequality (see [17, Lemma 2.1])

$$
\sum_{l=1}^{n-1}(n-l)|r(l)| \leq \frac{C n^{2-\gamma}}{(1-\gamma)(2-\gamma)},
$$

so we get the bound

$$
\frac{1}{n^{2}} \sum_{j=1}^{n} \sum_{k=1}^{n}\left|E_{\eta} \eta_{j} \overline{\eta_{k}}\right| \leq \frac{\sigma_{\eta}^{2}}{n}+\frac{2 C}{n^{\gamma}(1-\gamma)(2-\gamma)},
$$

which is again applicable in Lemmas 3.1 and 4.1. Consequently, relevant results on asymptotic consistency and finite sample properties, similar to Theorems 4.1-4.3, can also be formulated for the above defined short and long range dependent observation errors model.

6. Conclusions. This work is a continuation of the author's previous investigations on finite sample and asymptotic properties of orthogonal series regression estimation for irregular observation point designs [16]. Statistical and asymptotic aspects of estimators based on the standard DFT coefficients were also examined in the author's earlier works [14, [15], [18. This time the least squares method is used to estimate the Fourier coefficients of a complex valued regression function observed at unevenly sampled times. Determination of such estimators from available observations can be recognized as discrete spectral analysis of unevenly spaced data.

Similar discrete spectral or spectral-temporal analysis of a real valued regression function $f \in L^{2}[0,1]$ can be performed in an analogous way using the orthonormal systems of Haar functions or Walsh functions in the Hilbert space $L^{2}[0,1]$ [26].

Even in the case of observation errors with non-zero mean values $E_{\eta} \eta_{j}=$ $\delta_{j} \neq 0, j=1, \ldots, n$, the properties of the Fourier coefficient estimators ensure that the transformation of the observation vector $y=\left(y_{1}, \ldots, y_{n}\right)^{T}$ into the vector $\hat{c}^{N}$ is a continuous linear operation, since $\left\|\hat{c}^{N}\right\| \leq C(n, \alpha)\|y\|$ for $N=2 m+1, m \leq[n \alpha /(8 \pi)]$, where $0<\alpha \leq[n /(8 \pi)] 8 \pi / n$ (see inequality (9)).

The results of this work can be easily extended to the case of analogous irregular designs on any iterval $[0, T]$, where $T>0$.

Observation times involving distortions, that is, $t_{j}=(j-1 / 2) / n+\xi_{j}, j=$ $1, \ldots, n$, where $\xi_{j}$ are i.i.d. random variables with e.g. uniform distribution on $(-1 /(2 n), 1 /(2 n))$, are sometimes used in estimation of regression functions and are called equispaced designs subject to jittering [19]. Other jittered 
sampling designs related to signal reconstruction are considered in [10]. Such designs are also applied to study the robustness of regression estimators to uncertainties in the independent variable [21].

Another approach to construction of projection estimators using trigonometric functions, the Gasser-Müller type regression estimator, is investigated in [6], 7], [21], 27]. The Fourier coefficient estimators used in that approach are obtained by some integration procedure involving function observations available at fixed times with bounded distortions, i.e. at $t_{j}=s_{0 j}+\xi_{j}$, $j=1, \ldots, n$, where $0 \leq s_{01}<s_{02}<\cdots<s_{0 n} \leq 1,\left|\xi_{j}\right| \leq C / n$ [21]. Hence, this work extends the results of [21] to the case of least squares projection estimation of regression. An analogous integration procedure is also applied in the case of random observation times $t_{j}, j=1, \ldots, n$, with probability density $\varrho \in L(0,1), \varrho \geq c>0$ [6], [7], 27]. The case of random designs of observation times and least squares projection estimators based on trigonometric functions was earlier examined in [1, so it was not considered here. However, if we assume that $f \in L^{2}[0,1]$ satisfies a certain smoothness condition, e.g. is $\alpha$-Hölder with exponent $0<\alpha \leq 1$, as in [6], then using Theorems 4.2 and 4.3 one can also estimate convergence rates of the average sample error and the IMSE error in the case of observation times design considered here. Moreover, under the same condition on $f$, using Lemmas 3.1 and 3.2 which give upper bounds on variance and squared bias of the least squares Fourier coefficient estimators, it is possible to obtain pointwise mean square error convergence rate of the corresponding projection estimator, similarly to 6 .

Acknowledgements. This research work was supported by the Polish National Science Centre through the grant No. UMO-2014/13/B/ST10/ 04975 carried out at the Space Research Centre of Polish Academy of Sciences.

\section{References}

[1] A. Cohen, M. A. Davenport, D. Leviatan, On the stability and accuracy of least squares approximations, Found. Comput. Math. 13 (2013), 819-834.

[2] S. Ferraz-Mello, Estimation of periods from unequally spaced observations, Astronom. J. 86 (1981), 619-624.

[3] G. Foster, The cleanest Fourier spectrum, Astronom. J. 109 (1995), 1889-1902.

[4] G. Foster, Time series by projection I: statistical properties of Fourier analysis, Astronom. J. 111 (1996), 541-554.

[5] C. Gasquet and P. Witomski, Fourier Analysis and Applications-Filtering, Numerical Computation, Wavelets, Springer, New York, 1999.

[6] W. Greblicki and M. Pawlak, Dynamic system identification with order statistics, IEEE Trans. Information Theory 40 (1994), 1474-1489. 
[7] W. Greblicki and M. Pawlak, Nonparametric System Identification, Cambridge Univ. Press, New York, 2008.

[8] J. Koh and T. K. Sarku, Spectral analysis of non-uniformly spaced data using least squares method, Digital Signal Processing 15 (2005), 44-55.

[9] N. R. Lomb, Least-squares frequency analysis of unequally spaced data, Astrophys. Space Sci. 39 (1976), 447-462.

[10] E. Masry, Analysis of signal reconstruction with jittered sampling, IEEE Trans. Signal Processing 59 (2011), 27-34.

[11] A. Mathias, F. Grond, R. Guardans, D. Seese, M. Canela and H. H. Diebner, Algorithms for spectral analysis of irregularly sampled time series, J. Statist. Software 11 (2004), no. 2, 1-27.

[12] H. Niederreiter and A. Winterhof, Applied Number Theory, Springer, Cham, 2015.

[13] S. D. Pagiatakis, Stochastic significance of peaks in the least squares spectrum, J. Geodesy 73 (1999), no. 2, 67-78.

[14] W. Popiński, On consistency of discrete Fourier analysis of noisy time series, Artificial Satellites J. Planetary Geodesy 32 (1997), no. 3, 131-142.

[15] W. Popiński, Least-squares trigonometric regression estimation, Appl. Math. (Warsaw) 26 (1999), 121-131.

[16] W. Popiński, Orthogonal series regression estimators for an irregularly spaced design, Appl. Math. (Warsaw) 27 (2000), 309-318.

[17] W. Popiński, Orthogonal series regression estimation under long-range dependent errors, Appl. Math. (Warsaw) 28 (2001), 457-466.

[18] W. Popiński, Trigonometric regression estimation for observations with additive and multiplicative errors, Comm. Statist. Theory Methods 45 (2016), 804-812.

[19] R. Porto, P. Morettin, D. Percival and E. Aubin, Wavelet shrinkage for regression models with random design and correlated errors, Brazilian J. Probab. Statist. 30 (2016), 614-652.

[20] E. Rafajłowicz, Nonparametric least-squares estimation of a regression function, Statistics 19 (1988), 349-358.

[21] E. Rafajłowicz, Non-parametric identification with errors in independent variables, Int. J. Systems Sci. 25 (1994), 2031-2038,

[22] C. R. Rao, Linear Statistical Inference and its Applications, Wiley, New York, 1973.

[23] W. Rudin, Functional Analysis, McGraw-Hill, New York, 1991.

[24] J. D. Scargle, Studies in astronomical time series analysis II-Statistical aspects of spectral analysis of unevenly spaced data, Astrophys. J. 263 (1982), 835-853.

[25] R. C. Singleton, An algorithm for computing the mixed radix fast Fourier transform, IEEE Trans. Audio Electroacoustics 17 (1969), 93-103.

[26] D. S. Stoffer, Walsh-Fourier analysis and its statistical application, J. Amer. Statist. Assoc. 86 (1991), 461-485, .

[27] P. Śliwiński, Fast algorithms for non-linearity recovering in Hammerstein systems with ordered observations, in: Proc. 10th IEEE Int. Conference on Methods and Models in Automation and Robotics MMAR 2004, 451-456.

[28] P. Vaniček, Approximate spectral analysis by least-squares fit, Astrophys. Space Sci. 4 (1969), 387-391.

[29] P. Vaniček, Further development and properties of the spectral analysis by leastsquares, Astrophys. Space Sci. 12 (1971), 10-33.

[30] K. Yosida, Functional Analysis, Springer, New York, 1980, .

[31] A. Zygmund, Trigonometric Series, Vols. I and II, Cambridge Univ. Press, Cambridge, 1977. 
Waldemar Popiński

Space Research Centre

Polish Academy of Sciences

Bartycka 18a

00-716 Warszawa, Poland

ORCID 0000-0001-9585-1040

E-mail: w.popinski@stat.gov.pl 